dann dürften wir wohl auch ein großes Stück in der Erkenntnis des Problems der künstlichen Ernährung weitergeirommen sein.

Für den Nutzen der Frauenmilch hat man aber nicht nur die chemischen und physikalischen Unterschiede der beiden Milchen angeführt, sondern die spezifisch biologischen Eigenschaften. Insbesondere hat man den Vorzug der Frauenmilch in dem Vorhandensein gewisser Fermente und anderer spezifischer Stoffe gesehen, die zum Teil koktostabil, meistens aber sehr unbeständig gegen Hitze sind, und wie es scheint selbst bei unpfleglicher Behandlung der Milch zugrunde gehen können. Dem an der Brust trinkenden Kind kommen sie in vollem $\mathrm{Maße}$ zugute, fraglich ist es schon, ob das aus der Flasche die Frauenmilch trinkende Kind eine völlig gleichartige Nahrung in diesem Sinne erhält. Daß diese Stoffe der Kuhmilch, selbst wenn sie roh genossen werden, für den Säugling auch nicht entternt die gleiche Bedeutung haben wie die der Frauenmilch, braucht nicht betont $z \mathfrak{u}$ werden. Manche Stoffe, wie z.B. die sog. akzessorischen Nährstoffe, die das Wachstum in verschiedener Hinsicht spezifisch beeinflussen, scheinen in der Kuhmilch in gleicher Weise vorhanden $\mathrm{zu}$ sein. Viele andere kommen in der Kuhmilch nicht zur Geltung, da sie ,artspezifisch"“ gebildet sind. Man denke an Immunstoffe u. a. Wir möchten ganz allgemein die Bedeutung dieses Anteils der Brusternährung recht hoch einschätzen, aber die oft daraus abgeleitete Forderung, dem Kinde rohe Milch zu geben, ist müßig, denn auch rohe Kuhmilch bleibt artfremde Nahrung und die meisten dieser Nährstoffe sind sicher für die eigene Art biologisch so abgestimmt; daß sie in fremder Nahrung dargereicht, dem Kinde nicht den gleichen Ersatz bringen. Für die Nutramine scheint dies allerdings nicht der Fall zu sein; aber damit sind nicht etwa alle Ergänzungsstoffe erschöpft. Klinisch ist übrigens kein Vorzug der rohen Milch vor der gekochten erwiesen: Im Gegenteil sprechen klinische Erfahrungen gegen rohe Milch, zumal das Casein in der rohen Milch viel gröber gelabt und dadurch auch schlechter ausgenutzt wird (Caseinbröckel im Stuhl). In vielen schweren Krankheitsfällen sieht man immer wieder den durchschlagenden Nutzen der Frauenmilch, wobei wir aber nicht etwa den Eindruck haben, daß es nur die korrelativen Nährstoffverhältnisse von Eiweiß, Fett und Zucker usw. sind, die nützlich wirken. Gibt man z. B. Kuhmilchmischungen in annähernd fettgleichen Mischungsverhältrissen der einzelnen Nährstoffe, so bleibt oft jede Reparation aus. Ja auch bei der Frauenmilchdarreichung kann der Durchfall weiter bestehen, ja oft wieder stärker werden und doch sehen wir eine Reparation des Kindes eintreten. Das können wir wohl nur durch einen positiven Nutzen der Frauenmilch erklären und nicht etwa allein dadurch, daß diese Nahrung günstig auf den pathologischen Ablauf der Darmvorgänge wirkt. Diese können wir oft ebenso gut, ja öfter noch besser durch gewisse künstliche Nahrung beeinflussen; (Eiweißmilch) und doch erfolgt die Gesundung nicht so rasch. Es müssen wohl noch ganz besonders "heilende Stoffe" in der arteigenen Nahrung für das junge Kind vorhanden sein.

Endlich hat man den Keimreichtum in der Kuhmilch als die Ursache für den wechselnden Erfolg der künstlichen Ernährung angesehen, wobei wir nur an die Saprophyten der Milch denken. Das bakteriologische Milchproblem ist hier nicht zu behandeln. Das aber darf hier ausgesprochen werden, daß es für den Erfolg der künstlichen Ernährung wohl nicht die Bedeutung besitzt, die ihm von mancher Seite heute noch zuerkannt wird, wenn auch zugegeben werden soll, daß darüber das letzte Wort noch nicht gesprochen ist.

Wenn immer wieder darauf hingewiesen wird, daß für den Erfolg der künstlichen Ernährung nicht allein das Nahrungsproblem maßgebend sei, sondern ebenso konstitutionelle und konditionelle Mängel des Kindes in Betracht kämen, Dinge, die letzten Endes celluläre Probleme darstellen und daher experimenteller Forschung außerordentlich schwer zugänglich sind, so ist das richtig und natürlich und auch von groBer Bedeutung; aber streng genommen hat dies mit dem eigentlichen Problem der künstlichen Ernährung nichts zu tun, da dieser Faktor ganz ebenso bei der Brusternährung zuin Aus- druck kommt und sich vielleicht nur bei unnatürlicher Ernährung deutlicher zu erkennen gibt.

Zusammenfassend ist also etwa folgendes zu sagen: Eine eindeutige Beantwortung der Frage, warum der Erfolg der unnatürlichen Ernährung ein so viel unsicherer ist, ist nicht zu geben, ja sie wird sicher niemals zu geben sein, da hier eine Artspezifität der Brustmilch vorwaltet; die wir nicht zu meistern vermögen. Der Erfolg der künstlichen Ernährung wird abhängig sein von dem Verhältnis der Leistungsfähigkeit des Kindes zur Inanspruchnahme der zugeführten Nahrung. Dabei ist die Leistungsfähigkeit nicht nur in der angeborenen Konstitution begründet, sondern dauernd wechselnden Einflüssen unterlegen (konditionale Faktoren im Sinne TANDLERS). Der Versuch, die Schäden der zugeführten künstlichen Ernährung $z u$ vermindern, ist heute uber eine gesunde Empirie noch nicht hinausgekommen, wenn auch eine gewisse Anpassung der Kuhmilch an die Frauenmilch für den gesunden Säugling wohl von Vorteil ist.

\section{DAS PROBLEM DER RÖNTGENTIEFENTHERAPIE VOM PHYSIKALISCHEN STANDPUNKT ${ }^{1}$ ).}

\author{
Voñ
}

Prof. Dr. Friedrich Dessauer,

Direktor des Universitătsinstituts für physikal. Grundülagen d. Medizin, Frankỉ̛urt a. M

Wenige Dinge sind nach so langer Zeit ihrer Prüfung I8 Jahre nach den ersten Veröffentlichungen - so umstritten in den Meinungen der Kliniker als die Tiefentherapie de Carcinoms und anderer maligner Tumoren mit Röntgenstrahlen. Es fìnden sich radikale Ansichten, Ablehnung der Tiefentherapie als erfolglos, es gibt Kliniker, die behaupten; noch keinen einzigen röntgenologisch geheilten Fall von Carcinom gesehen zu haben, es gibt entgegengesetzt extreme Ansichten, welche der Bestrahlung in allen oder den meisten Fällen den Vorrang vor der Operation zuerkennen wollen; und es gibt dazwischen alle Schattierungen in der Ansicht über die Teilung der Therapie zwischen Operation und Bet strahlung.

Eine solch verworrene Lage $\mathrm{muB}$ einen besonderen Grund haben. Etwas muß an der Tiefentherapie mit Röntgenstrahlen sein, sonst wäre sie in den I8 Jahren ihres Bestehens als untauglich erkannt worden. Sie nimmt aber täglich breiteren Raum ein, und wer Lokalbefunde nach Bestrahlung gesehen hat, kann sich der Ansicht einfach nicht verschließen, daß hier wirklich eine gewaltige Waffe im Kampfe gegen maligne Tumoren vorliegt. Aber es geht eben so sicher aus dem jetzigen Zustande hervor, da 3 die richtige Handhabung der Tiefentherapie noch fehlt, sonst wären die Widersprüche unerklärlich und die überaus zahlreichen Versager. Auf dem letżten Kongre $B$ der amerikanischen Röntgengesellschaft Ende September I92I hörte ich die amerikanischen Radiologen berichten, welche im vergangenen Jahre herübergesandt worden sind, um die deutschen Resultate in der Tiefentherapie $\mathbf{z a}$ studieren. Sie sagten hauptsächlich zweierlei: Daß sie in einigen Kliniken an selbst untersuchtem Krankenmatetial erstaunliche Resultate, auch weit zurückliegende Resultate, gesehen hätten, und daß man bei uns in Deutschland : viel weiter sei wie bei ihnen; zweitens aber sagten sie, hinsichtlich der deutschen Methode seien sie in Verlegenheit, denn in fast jeder Klinik würde es anders gemacht, jeder hätte seine eigene Methode; und wenn auch gewisse Richtlinien gemeinsam wären, so wüßten sie bei dem Widerstreit der Meinungen nicht, welche Methode sie empfehlen sollen.

Der zweite Gedanke beleuchtet die Lage. Wo in der Naturwissenschaft hundert verschiedene Methoden zum selben sehr schwer erreichbaren Ziel nebeneinander laufen, da fehlt die Methode. So verschwenderisch ist die Natur nicht, da $B$ sie die Heilung eines ausgebreiteten Cärcinoms mit einem physikalischen Medikament auf sehr mannigfache Art zulassen könnte. Gèwöhnlich hat die Natur nưr eine Lösung, vielleicht

1) Nach einem Vortrage, gehalten am 22. Februar 1922 in Verein der Arzte Wiesbadeus. 
mit ein paar untergcordneten Variationen. Wo aber die Variation so groß erscheint, ist $\mathrm{zu}$ befürchten, daß sehr viele Fehler unterlaufen, und man muß die Grundlagen prüfen.

Bei der Therapie mit einem physikalischen Medikament hat man es mit zwei Problemen zu tun, dem physikalischen und dem biologischen. Zweifellos ist das biologische Problem, die Art, die Richtung und Stärke, wie Zellen auf das Röntgenlicht reagieren, welches sie einhüllt, auch wenn sie in der Tiefe liegen, von seiner Lösung weit entfernt. Das vor dem biologischen ruhende physikalische Problem ist aber in der letzten Zeit sehr weit gediehen. Man kann sagen, es ist im wesentlichen gelöst. Gelöst ist das Problem der lokalen physikalischen Dosierung: Man kann qualitativ und quantitativ einigermaßen genau sagen, welcher physikalische Einfluß an irgendeiner Stelle einer durchstrahlten Zone, also auch in der unzugänglichen Tiefe des Körpers zur Wirkung kommt, und man hat darüber neuerdings Methoden, die zwar mühselig sind, aber gestatten, jeder beliebigen. Stelle in der Tiefe ein vorher überlegtes Ausmaß des physikalischen Einflusses zuzuführen, die einverleibte Dosis also so zu verteilen, daß sie hinreichend groß und doch wiederum nicht zu groß und darum nicht schädlich ist. Angesichts dieser Tatsache, daß die Physik neuerdings solche Möglichkeiten erschlossen hat, liegt die Überlegung nahe, damit durchzuprüfen, ob das vielfache Versagen der Tiefentherapie auf physikalische Fehler zurückgeführt werden könnte. Nicht als ob ich die Ansicht aussprechen wollte: wenn alle physikalischen Fehler vermieden würden, würde das Carcinom mit der Tiefentherapie heilbar sein. Darüber kann man heute nichts aussagen, man kann höchstens eine Hoffnung geben. Zwar Klarheit wird man erst bekommen, wenn man die physikalischen Bedinggungen der Tiefentherapie richtig macht, so daß wenigstens diese Voraussetzung für eine Heilungsmöglichkeit gegeben ist. Wird aber das Physikalische falsch gemacht (also gewisse Teile der Erkrankungszone nicht oder unzureichend bestrahlt oder erhalten sie eine zureichende Dosis nur durch eine schwere Schädigung, durch Uberbelastung des Körpers mit dem Medikament), dann kann man überhaupt nicht erfahren, ob die Tiefentherapie ein gutes Mittel gegen solche maligne Neubildungen ist oder nicht.

Sind unter den zahlreichen Versagern sehr zahlreiche aus physikalisch unrichtiger Methodik? Wenn ja, so werden die biologischen Schlüsse schwer. Kann man die physikalische Dosierung aber als richtig ansehen, so sind die Versager und die guten Resultate - biologisch auszuwerten. Mit den gegenwärtigen Mitteln der Physik läßt sich hinreichend genau nachprüfen, ob bei den bekannten in Zeitschriften und Büchern veröffentlichten Methoden das Medikament physikalisch richtig verabfolgt wird und ob überhaupt Möglichkeiten der Heilung bestehen. Daß, - wie kürzlich gesagt wurde (Dtsch. med. Wochenschr. 47, H. I2, S. 386), die physikalische Dosierung in den verschiedenen Fällen und Kliniken einigermaßen gleich sei (weil Apparate, Röhren, Filter ähnlich ođer gleich sind) - daran ist, wie quantitative Uberlegung zeigt, auch im Entferntesten nicht zu denken. Dieses Problem: ist man soweit, Versager sicher biologisch zu deuten, weil die physikalischen Bedingungen richtig sind - oder umgekehrt sind die physikalischen Bedingungen so unvollkommen, da $\beta$ man häufig gar kein Resultat erwarten darf - dieses Problem soll Gegenstand der folgenden Betrachtung sein.

SEIrz formulierte seine Ansicht kürzlich (Münch. med. Wochenschr. I92I, Nr. 35, S. IIO9) am AbschluB einer Arbeit über "Carcinomgenese und Carcinomdosis" in den Worten: ,Es läuft daher das Problem, ob es möglich ist, ein Carcinom durch Röntgenstrahlen zu keilen, im wesentlichen und letzten Endes auf die Möglichkeit einer exakten Dosierung hinaus." Diese Ansicht wird zur Zeit stark angefochten. Daß sie aber eine sehr große Wahrheit enthalten kann, zeigt die physikalische Uberlegung evident.

Selbstverständlich müssen einige biologische Grundannahmen auch für die physikalische Betrachtung gemacht werden. Es sind dies die bekannten Annahmen, daB Zellen auf Strahlen reagieren, daß sie nach ihrer biologischen Verschiedenheit verschieden reagieren, daß es möglich ist, mit ganz bestimmten, nicht zu kleinen, nicht zu großen Dosen des Strahlenmedikamentes pathologische Zellen irgendwie (wie kann dahingestellt bleiben, ob Gonoceptorenschädigungen oder direkte Zellentötung oder nur Herabminderung der Vitalität, so daß dann die natürlichen Kräfte des Körpers ausreichen) zu schädigen, daß die Schädigung zunächst da auftritt, wo die Strahlung gewirkt hat, wie man ja histologisch sieht und was keineswegs bedeutet, da $B$ indirekte Wirkung, „Fernwirkungen" nicht auch möglich sind. Diese Annahmen werden wohl von allen Fachleuten zugestanden und darauf baut die Physik auf und schließt:

I. Es muß im ganzen Bereiche der Erkrankungen, wo also pathologische Zellformen erwartet werden, eine für die dortliegenden Zellen hinreichende Dosis des Medikamentes wirksam sein. Ist sie nur in einem Teil wirksam, so ist wahrscheinlich, sogar sicher, daß der dem direkten Strahleneinfluß nicht oder unzureichend ausgesetzte Teil des Herdes nicht geheilt wird (oder geschädigt wird, Reizwirkung?).

2. Die Physik schließt weiter: Diese hinreichende Dosis des Medikamentes, die man vorausbestimmen muß, darf nirgends so groß sein, oder durch eine ungeeignete Methode so groß werden, đaß sie - auch durch indirekte Wirkungen ernsthafte Körperschädigungen herbeiführt.

Sieht man sich die jetzt gebräuchlichen Methoden an und prüft sie nach mit den Hilfsmitteln der Gegenwart, so kommt man zu dem sehr bedrückenden Eindruck, daB nur in seltenen Fällen mit diesen jetzt zumeist angewandten Methoden diese beiden Bedingungen erfüllt werden. Die Empfindung, daß es so sei, habe ich schon lange. Nachprüfung einzelner Fälle schafft darüber traurige Gewißheit und die Pflicht es auszusprechen: Das übliche Vorgehen ist gewöhnlich so: Die erkrankte Zone, z. B. ein nicht melir operierbares Brustcarcinom wird in fast jeder Klinik wesentlich verschieden - nach irgendeiner Regel mit 2, 3 oder mehreren Feldern von vorne, von hinten, von der Seite, mit größeren oder kleineren Abständen, mit sehr harten oder weniger harten Strahlen behandelt. Es müßte doch sehr sonderbar zugehen, wenn bei all den hundert Modifikationen, die hierdurch gegeben sind, wirklich in jedem Falle in der ganzen Ausdehnung des Carcinoms überall eine gerade hinreichende, also vielleicht überall eine ungefähr gleichgroße (homogene) Dosis und nirgends dabei eine zu große, schädigende Dosis einverleibt würde. Wenn es dann manchmal oder sogar ziemlich oft gut geht, mag eine hohe. Empfindlichkeit der pathologischen Zellen, eine starke Beteiligung der Abwehrkräfte des Körpers geholfen haben. Wenn es aber in der überwiegend großen Anzahl der Fälle nicht dauernd gut geht, so kann man eigentlich meistens beweisen (wenn die Bestrahlungsbedingungen genau aufgezeichnet wurden), daß das Medikament an verschiedenen Stellen ganz unzureichend gewesen ist so daß die Heilung nicht erwartet werden konnte. Um diese unzureichende Dosierung zu vermeiden, griff man im Laufe der Zeit - und in manchen Fällen mit Erfolg - zu dem Aushilfsmittel maximaler Dosen. Aber dabei tritt die Gefahr, durch das Heilmittel Unheil anzurichten, so stark auf, daß man es nicht allgemein so machen könnte.

Zwei entscheidende Fortschritte der Physik sind im Laufe der letzten Zeit zu Hilfe gekommen und gestatten eine physikalisch exakte Dosierung und Methodik. Der eine Fortschritt ist der: Extrem harte Strahlen - schon wegen ihrer Verteilung das einzig aussichtsreiche Medikament aus den mit dem Sammelnarnen Röntgenstrahlen bezeichneten untereinander in ihren Eigenschaften sehr verschiedenen Wellenarten - die wir schon seit Jahren erzeugen konnten, können wir mit Sicherheit, Gleichmäßigkeit, in hinreichender Menge unter hinreichend genauer Messung hervorbringen, und zwar dieselben Strahlen mit allen möglichen Maschinen an allen möglichen Orten. Die physikalischen Eigenschaften dieser Strahlen sind bekannt, und solange man bekannte Strahlen verwendet, also die Qualität seines Medikamentes mißt, kennt man diese Eigenschaften. Diese Röntgenstrahlen einer hinreichend genau gemessenen Art breiten sich im Körperinnern aus, unsichtbar zwar für das Auge, aber in Wirklichkeit hüllen sie die durchstrahlte Körperzone in eine Lichtatmosphäre, so 
daß die durchstrahlte Zone des Körpers, könnten wir Röntgenstrahlen sehen, sich ausnähme wie eine leuchtende weiße Wolke oder ein von Lichtstrahlen durchwandertes, etwas getrübtes Glas. Irgendwo in dieser Lichtstraße befindet sich an einem Orte eine bestimmte Helligkeit. Von Ort zu Ort ist diese Helligkeit verschieden und damit der Maßstab des Einflusses, die Dosis. Bei der Wanderung eines Lichtkegels sind Unterschiede von I : 30 in der Helligkeit an den verschiedenen Orten allgemein vorhanden, insbesondere große Unterschiede zwischen den zentralen Teilen des Lichtweges und dem peripheren Teile, dem der Lichtquelle näheren und dem ihr entfernteren. Was man nun früher nicht wuBte, aber heute wei $B$, das ist, wie diese Lichtverteilung in einem Kegel, der den Körper durchwandert, wirklich ist, und zwar unter den verschiedensten Bedingungen: Bei verschiedenen Strahlenarten, verschiedenen Abständen, Feldgrößen. Für eine große Zahl von solchen Fällen ist die Lichtverteilung in einer jahrelangen Laboratoriumsarbeit gemessen (nicht berechnet) worden. Diese Verteilung kannte man früher nicht, und man hatte keinerlei Sicherheit, ob eine pathologische Zelle irgendwo im bestrahlten Gebiet der halben, doppelten oder dreifachen Lichtintensität ausgesetzt war. Vielleicht hat man im allgemeinen zu viel bestrahlt, sicher an vielen Orten des durchstrahlten Gebietes trotzdem zu wenig. Über die Messungen, ihre Methoden und Ergebnisse kann hier nicht berichtet werden. ${ }^{1}$ ) Diese Meßarbeit gestattete die schon erwähnte Nachprüfung der früher angewandten Methode und zeigte in der Tat, daß bessere Resultate, als sie vorliegen, schon aus physikalischen Gründen nicht erwartet werden können. Da die einzelnen Bestrahler ja nicht wissen konnten, wieviel von jedem einzelnen dieser Felder an jeden einzelnen Punkt in der Tiefe von dem Medikament hinkam - Messungen konnten höchstens an einer oder zwei Stellen in einer Körperhöhle in der Tiefe gemacht werden, aber die seitliche Verteirung der Energie entzog sich der Kenntnis - so war es eine Zufallssache, wenn die richtige Verteilung des Medikamentès die Voraussetzung zu einer Heilung bot. Denn das ist der große, die erheblichsten Schwierigkeiten bereitende Unterschied eines solchen physikalischen Medikamentes, daß es räumlich wirkt, daß raumgeometrische Betrachtungen in die Röntgenstrahlendosierungen als eine medizinisch fremde Vorstellung hineinkommen. Nunmehr lassen sich auf Grund der Intensitätsverteilungsmessungen Methoden aufbauen, physikalisch richtig zu bestrahlen, das Medikament überall zureichend, nirgends in zu großer Dosis davon hinzubringen, wo es wirken soll, und fernzuhalten, wo es nicht wirken soll. Diese Methoden sind gewiß nicht leicht, aber sie sind möglich, sie sind in der Entwicklung. Sie lassen bei weitem nicht mehr die Variationen in der Therapie zu, die jetzt bestehen, und welche die amerikanischen Berichterstatter in Erstaunen setzten. Man findet oft durch Uberlegung eines Falles, daß Lage und Form eines Krankheitsherdes überhaupt nur eine Möglichkeit gestattet, ihn hinreichend $\mathrm{zu}$ bestrahlen ohne irgendwo zu schädigen, ohne irgendwo $\mathrm{zu}$ wenig zu geben. Es wird Zeit vergehen, bis diese Methoden genügend entwickelt sind und bis sie an vielen Orten richtig angewandt werden. Dann aber wird man in đer Lage sein, die biologischen Veränderungen an Zellen immer wieder - auch in beliebiger Tiefe - auf bekannte Dosen des physikalischen Einflusses zurückzuführen, und dann wird die Zeit kommen, wo man entscheiden kann, ob und wie weit die Tiefentherapie mit Röntgenstrahlen ein Heilmittel für maligne Tumoren ist oder nicht.

\section{ORIGINALIEN.}

\section{DIE ANWENDUNG HYPERTONISCHER TRAUBEN- ZUCKERLÖSUNGEN BEI ORGANISCHEN HERZ- ERKRANKUNGEN²). (Experimentelles und Klinisches.) Von \\ Felix Klewitz und Rudolf KirchHeIm.}

Aus der Medizinischen Universitätsklinik zu Königsberg i. Pr. (Direktor: Geh.-Rat Prof. Dr. MATTHES).

Die Behandlung gewisser Erkrankungen des Herzens mit hypertonischer Traubenzuckerlösung geht bekanntlich auf eine Anregung von BüDINGEN zurück. Die Vorstellung ist dabei die, daß bestimmte Herzerkrankungen, die BüDINGEN als ,Kardiodystrophien" bezeichnet; durch einen zu niedrigen Gehalt des Blutes an Traubenzucker bedingt sein können. Die Anwendung hochprozentiger Zuckerlösung ist seither vielfach angewandt worden und zum Teil auch anscheinend mit gutem Erfolg; freilich fehlt es auch nicht an Ablehnungen. Ehe wir selbst an eine Nachprüfung gingen, lag uns daran, die klinischen. Versuche auf eine experimentelle Basis zu stellen. Wenn auch im Tierexperiment erhaltene Resultate nicht restlos auf den menschlichen Organismus übertragbar sind, so glauben wir doch, zeigen zu können, daß die im Tierexperiment gewonnenen Erfahrungen in mancher Beziehung sich mit denen am Krankenbett decken.

Unsere Versuche wurden an dem nach dem LANGENDoRFschen Verfahren überlebend gehaltenen. Warmbiüter(Kaninchen-) Herzen ausgeführt.

Dabei wird bekanntlich eine Kanüle in die Aorta eingebunden, so daß die Ernährung des Herzmuskels durch das Coronargefäßsystem erfolgt. Wir haben in jedem Fall vorher das Tier gänzlich entblutet und durch Ringerlösung blutfrei gespült. Die Herz-

y) Vgl. hierüber DESSAUER u. VIERHELLER, Physikal. Zeitschr. 21, I7r. I9zo. Zeitschr. f. Physilk Igzo, H. x. - Strahlentherapie Bd. XII. - DESSAUER: Zur Therapie des Carcinoms mit Röntgenstrahlen, Steinkopf, Dresden roz2. - DESSAUER: Dosierung von Röntgenstrahlen vom physikalischen Standpunkt (bei Steinkopf im Erscheinen begriffen). - JARRE u. POHLE: Methodik der Röntgen-Tiefentherapie Erscheinen begriffen). - JARRE u. POHLE: Methodik der Röntgen-Tiefentherapie
des Uterus- und Brustcarcinoms vom physikalischen Standpunkt (bei Steinkopf, do.). 2) Nach einem Vortrag im Verein für wissenschaftliche Heilkunde, Königsberg i. Pr. kontraktionen wurden auf ein Kymographion aufgezeichnet, und zwar durch einen Hebel, auf den die Herzbewegungen durch eine Fadenverbindung übertragen wurden. Es kam nur ganz ausnahmsweise vor, daß die Wiederbelebung des Herzens nicht oder nicht einwandfrei gelang. Die Ernährung des Herzens erfolgte durch Ringer- oder häufiger durch Lockelösung; der Ausfall der Versuche blieb durch die Wahl der Nährlösung unbeeinflußt. Nachdem die unregelmäßigen und in ihrer Intensität häufig sehr schwankenden Kontraktionen in der ersten Periode nach gelungener Wiederbelebung vorüber waren, wurde eine Kurve aufgenommen; war diese gleichmäßig, so erfolgte eine Injektion von $1 / 2 \mathrm{ccm}$ einer 30 proz. Traubenzuckerlösung, und zwar in das Schlauchende dicht vor seinen Ansatz in die Aortenglaskanüle. Etwa von Minute zu Minute, bisweilen auch in etwas längeren Abständen, wurde wieder ein Kurvenstück aufgenommen. Die Menge von $1 / \mathrm{ccm}$ wurde gewählt, weil sie etwa dem Herzschlagvolumen eines mittleren Kaninchenherzens entspricht. Wir haben am Menschen, wie im klinischen Teil noch ausgeführt wird, mit wenigstens schätzungsweise analogen Mengen gearbeitet.

Auf diese Weise haben wir Versuche an I9 Tieren angestellt und verfügen über einige Dutzend Kurven. Das Ergebnis unserer Untersuchung läBt sich mit folgenden kurzen Worten zusammenfassen: In der überwiegenden Mehrzahl der Fälle nimmt die Höhe der Kontraktionen fast unmittelbar nach der Injektion der hypertonischen Zuckerlösung zu. Diese Zunahme der Kontraktionsgröße ist meistens schon mit dem Auge erkennbar, auf der Kurve aber dann sehr deutlich. Hand in Hand mit der Zunahme der KontraktionsgröBe geht eine bessere Durchblutung des Herzmuskels. Zählt man die Tropfenzahl vor und nach der Injektion, so kann man eire mitunter nicht unerhebliche Zunahme derselben meistens unmittelbar nach der Einspritzung feststellen. Die Dauer der kontraktionssteigernden Wirkung der Zuckerlösung ist verschieden lang, gewöhnlich hält sie einige Minuten an. Durchschnittswerte lassen sich nicht angeben. Ganz ohne Ausnahmen ist diese Wirkung der hypertonischen Zuckerlösung nicht, aber die Zahl der Versager ist verschwindend klein. Es fällt auf, daß die Wirkung der Zuckerlösung besonders dann deutlich erkennbar ist, wenn das Herz anfängt zu erlahmen; in diesem Stadium kann ein etwa vorhandenes 